\title{
Low energy and high energy dumps for ELI-NP accelerator facility: rational and Monte-Carlo calculations' results
}

\author{
A. Esposito ${ }^{1}$, O. Frasciello ${ }^{1, \star}$, and M. Pelliccioni ${ }^{1}$ \\ ${ }^{1}$ Istituto Nazionale di Fisica Nucleare - Laboratori Nazionali di Frascati, Via E. Fermi 40, 00044 Frascati, Italy.
}

\begin{abstract}
ELI-NP will be a new international research infrastructure facility for laser-based Nuclear Physics to be built in Magurele, south west of Bucharest, Romania. For the machine to operate as an intense $\gamma$ rays' source based on Compton back-scattering, electron beams are employed, undergoing a two stage acceleration to $320 \mathrm{MeV}$ and $740 \mathrm{MeV}$ (and, with an eventual energy upgrade, also to $840 \mathrm{MeV}$ ) beam energies. In order to assess the radiation safety issues, concerning the effectiveness of the dumps in absorbing the primary electron beams, the generated prompt radiation field and the residual dose rates coming from the activation of constituent materials, as well as the shielding of the adjacent environments against both prompt and residual radiation fields, an extensive design study by means of Monte Carlo simulations with FLUKA code was performed, for both low energy $320 \mathrm{MeV}$ and high energy $720 \mathrm{MeV}(840 \mathrm{MeV})$ beam dumps. For the low energy dump we discuss also the rational of the choice to place it in the building basement, instead of installing it in one of the shielding wall at the machine level, as it was originally conceived. Ambient dose equivalent rate constraints, according to the Rumenian law in force in radiation protection matter were $0.1 \mu \mathrm{Sv} / \mathrm{h}$ everywhere outside the shielding walls and $1.4 \mu \mathrm{Sv} / \mathrm{h}$ outside the high energy dump area. The dumps' placements and layouts are shown to be fully compliant with the dose constraints and environmental impact.
\end{abstract}

\section{Introduction}

The Extreme Light Infrastructure - Nuclear Physics (ELINP) constitutes one of the three arms of the European Extreme Light Infrastructure project, presently being in the building process. Using an high intensity laser up to $10^{24} \mathrm{~W} / \mathrm{cm}^{2}$ and a high energy low bandwidth gamma beam system (GBS), it will be a new international research infrastructure facility for laser based nuclear physics. The facility will provide a broad range of science covering new nuclear physics, astrophysics, fundamental high field physics as well as applications in nuclear materials, radioactive waste management, material science and life sciences.

The ELI-NP project, as defined by its promoter and beneficiary, the Romanian National Institute of Nuclear Physics and Engineering "Horia Hulubei" (IFIN-HH), will consist of ground-breaking research systems equipped with a $2 \times 10 \mathrm{PW}$ laser system and a $740 \mathrm{MeV}$ (maximum operation energy) $e^{-}$linac, providing a tunable $0.2-19.5$ $\mathrm{MeV}$ GBS. The $\gamma$ rays are produced letting the relativistic $e^{-}$beam interact with a $2.4 \mathrm{eV}$ laser pulse at a point. The ELI-NP GBS will allow the production of $\gamma$ rays up to $\approx$ 19.5 MeV with a maximum $e^{-}$beam energy of $740 \mathrm{MeV}$.

An eventual energy upgrade of the $e^{-}$linac should raise the maximum energy up to $840 \mathrm{MeV}$. This operation mode has been used actually, in the prompt radiation

\footnotetext{
^e-mail: oscar.frasciello@lnf.infn.it
}

and activation simulations, in order to assess results for the most conservative scenario.

According to the Technical Design Reports (TDRs), finalized and approved by ELI-NP International Scientific Advisory Board in June 2015, the ELI-NP Center will have eight experimental rooms, some of them being depicted in figure 1, namely: E1 for laser induced nuclear reactions; E2 for nuclear resonance fluorescence applications; E3 for positron source; E4/E5 for accelerated particle beams induced by High Power Laser System (HPLS) $(0.1 / 1 \mathrm{PW})$ at high repetition rates; E6 for intense $e^{-}$and $\gamma$ beams induced by high power laser beams; E7 for experiments with combined laser and $\gamma$ beams; E8 for nuclear reactions induced by high energy $\gamma$ beams [1].

The design of both the low energy (320 MeV) and high energy $(840 \mathrm{MeV})$ line dumps has been worked out taking into consideration the main features of the systems, aiming to 1) avoid any interaction of the primary beam with the accelerator shielding walls, roof and floor included, 2) attenuate, at a reasonable cost and without compromising the utility of the apparatus for its design purpose, the prompt radiation at levels that are acceptable for humans outside the shielding walls, according with the Romanian law in force in radiation protection matter [2], 3) reduce the induced activity on the shielding walls, 4) reduce unwanted background in experimental detectors, 5) protect equipments against radiation damage and 6) reduce the cost of the shielding bulk. 


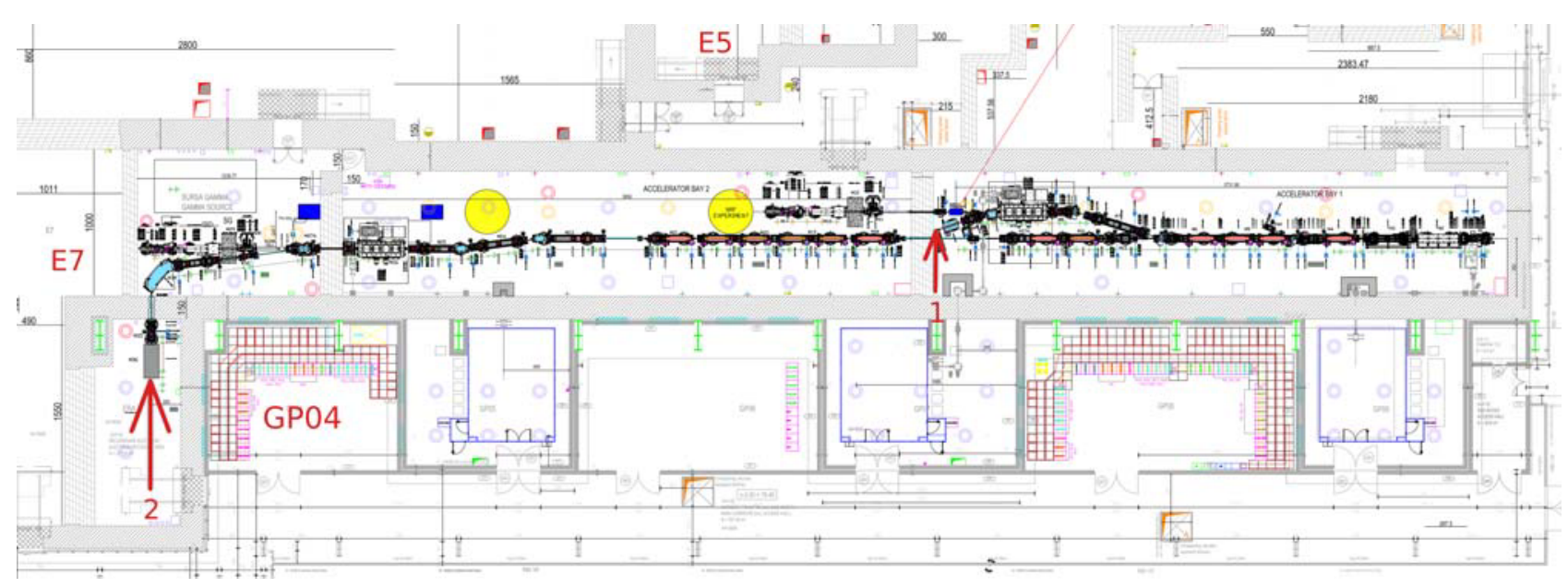

Figure 1. The ELI-NP machine layout and some experimental areas. Also indicated with red numbers are 1) the point where the low energy $320 \mathrm{MeV} e^{-}$beam is 90 degrees bended, in order to be delivered to the beam dump in the basement (as described in the text) and 2) the high energy $840 \mathrm{MeV}$ beam dump and its ERA host room (Courtesy of A. Falone and V. Pettinacci).

The ELI-NP shielding wall calculations have been performed based on the criteria that the dose uptake for professional exposed personnel must be only $10 \%$ of the legal limits, according to the Romanian law. In more detail, the individual effective dose must be less than $2 \mathrm{mSv} / \mathrm{y}$, the equivalent dose to the eye lens must be less then 15 $\mathrm{mSv} / \mathrm{y}$, the equivalent dose to the skin must be less then $50 \mathrm{mSv} / \mathrm{y}$ averaged over any $\mathrm{cm}^{2}$ area of the skin and the equivalent dose to the extremities such as hands, forearms, feet and ankles must be less then $50 \mathrm{mSv} / \mathrm{y}$ [3]. Moreover, the $\mathrm{H}^{*}(10)$ rate constraints dumps' design and shieldings must be compliant with, are $0.1 \mu \mathrm{Sv} / \mathrm{h}$ everywhere outside main shielding walls, as the doses to the members of the public, and $1.4 \mu \mathrm{Sv} / \mathrm{h}$ outside the high energy dump area, toward the GP04 room (refer to figure 1).

\section{Monte Carlo simulations}

All the simulations have been carried out with the FLUKA Monte Carlo code [4, 5], version 2011.2c. One of the major advantages of FLUKA, from the point of view of radiation safety applications, is the possibility to compute in the same simulation the ambient dose equivalent $\mathrm{H}^{*}(10)$ due to all the components of the prompt radiation field and to the time evolution of the activation products of irradiated materials, being the code able to transport their emitted radiation. The code, indeed, is able to solve analytically the Bateman equation, describing the time evolution of a system irradiated with a given pattern:

$$
\frac{\mathrm{d} N_{i}}{\mathrm{~d} t}=-\sum_{j \neq i}\left[\lambda_{j i}^{d}+\bar{\sigma}_{j i} \bar{\phi}\right] N_{i}+\sum_{j \neq i}\left[\lambda_{i j}^{d}+\bar{\sigma}_{i j} \bar{\phi}\right] N_{j}
$$

in which

$$
\bar{\phi}=\int \phi(E) \mathrm{d} E
$$

is the average spectrum of a particle fluence rate $\phi(E)$, which in equation 1 is kept constant during each time in- terval,

$$
\bar{\sigma}_{j i}=\frac{1}{\bar{\phi}} \int \phi(E) \sigma_{j i}(E) \mathrm{d} E
$$

is the particle induced cross section $\sigma_{i j}$, for the transmutation from isotope $i$ to isotope $j$, averaged over the spectrum and $\lambda_{j i}^{d}$ is the probability for radionuclide $i$ to decay into radionuclide $j$. FLUKA can then generate and transport the residual radiation, thus allowing the estimation of the residual dose rates due to the decay of the produced residuals.

As will be shown in the next section, the dumps' constituent material blocks, on which the primary particles do impinge, have dimensions exceeding the $320 \mathrm{MeV}$ and $840 \mathrm{MeV} e^{-}$range in the corresponding materials. For this reason those blocks must be treated as thick targets and, consequently, in our simulations the photo-nuclear physics was activated over the whole energy ranges for the relevant high $\mathrm{Z}$ elements in the dumps' models, namely $\mathrm{Pb}, \mathrm{W}, \mathrm{Cu}$ and concrete material.

In order to improve the statistics in the dose scoring regions, which are rather far apart from the interaction point of the primary particles of the beam with the concrete blocks inside the dumps (which are the first targets $e^{-}$do impinge upon), with several material regions in the middle, an importance biasing technique was used, increasing photons and neutrons importances up to a factor 512 toward the scoring regions. This way we were able to achieve calculated dose values affected by a statistical error not exceeding $10 \%$, whereas the calculated values for residual volume specific activities had an acceptable statistical error for all the scored radionuclides, kept at the level of few percent.

\section{Low energy $320 \mathrm{MeV}$ beam dump}

The low energy dump will be used to absorb the $320 \mathrm{MeV}$ $e^{-}$beam during the machine operation to produce the low energy $\gamma$ beam. The following assumptions subtend all the calculations for this dump: 
- $\mathrm{E}\left(e^{-}\right)=320 \mathrm{MeV}$

- $\mathrm{I}=1.6 \mu A \approx 9.9 \cdot 10^{12} e^{-} / s$

- Machine runtime= $3000 \mathrm{~h} / \mathrm{y}$.

As it was originally conceived, the low energy dump had to be placed at the accelerator level, horizontally arranged and mounted with additional concrete shields upon the building wall at the interface between accelerator bay 1 and 2 , as shown in the 3D model of figure 2. This choice, however, posed several problems to good practice criteria for the concepts of machine installation and operation. It resulted firstly from preliminary simulations that in order to achieve acceptable dose rates levels outside the lateral $1.5 \mathrm{~m}$ thick shielding walls, several tons of concrete shield had to be installed around the dump, for a suitable protection of personnel present in the adjacent control room against the prompt radiation [6]. This represented a structural issue in terms of additional load for the building wall. Secondly, the unshielded backscattered radiation, to which neutrons contributed mostly, could represent a severe constraint to sustainable machine runs, operations and components' radiation hardness. So we proposed (and the re-

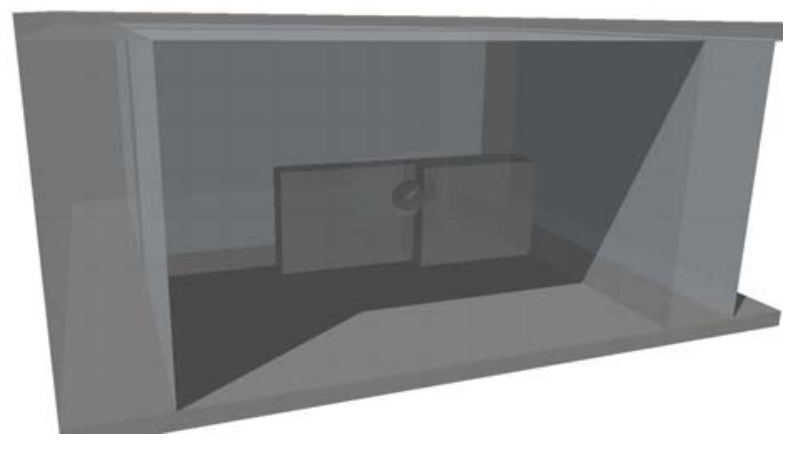

Figure 2. Low energy $320 \mathrm{MeV}$ beam dump placed in the accelerator bay, complete model with the needed lateral shields.

sults partially presented in this paper constituted the robust ground basis for the final acceptance of the proposal) to bend the low energy $e^{-}$beam at 90 degrees and to install its dump in the building basement area, under the accelerator floor, as shown in the 3D FLUKA model of figure 3, used for the simulations. Here the model is complete with the first eight nearest neighbours pillars, underground soil and water. The space sorrounding the dump is filled with air, with the standard composition of dry air at sea level. The dump itself 3D geometry is reported in figure 4 and does consist of a cylinder with diameter $150 \mathrm{~cm}$, height $70 \mathrm{~cm}$ and internal core of cylinder smaller blocks of ordinary concrete, tungsten and lead, sorrounded by lead and ordinary concrete. Finally it lies over an additional $50 \mathrm{~cm}$ of ordinary concrete stand, thus reaching an overall final height of $120 \mathrm{~cm}$.

\subsection{Prompt radiation field}

The interaction of the primary $e^{-}$beam with the dump materials produces secondary and tertiary radiations composed mainly of neutrons and photons. The computed

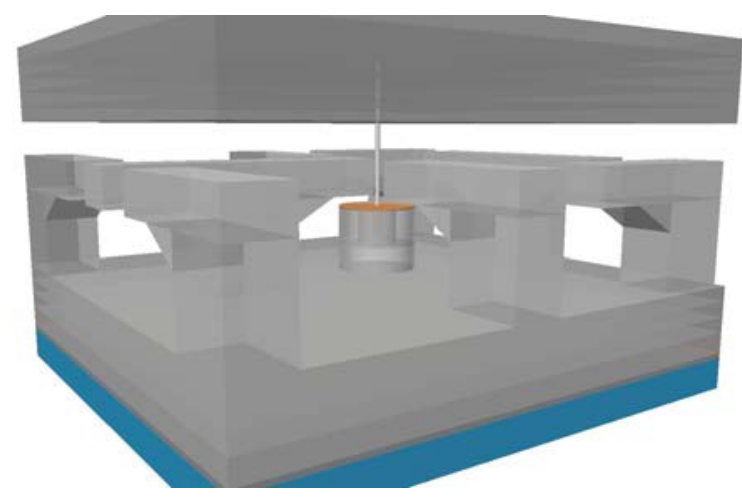

Figure 3. Low energy $320 \mathrm{MeV}$ beam dump placed in the building basement, complete model with ancillary nearest neighbours pillars, underground soil and water.

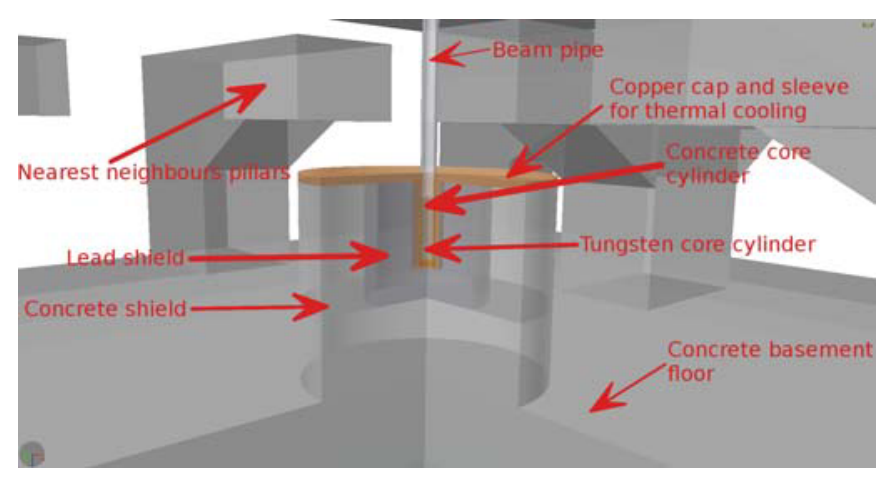

Figure 4. Low energy $320 \mathrm{MeV}$ beam dump internal structure detail (clipped view).

$\mathrm{H}^{*}(10) 3 \mathrm{D}$ distribution, in the building basement hosting the dump, due to the prompt radiation is reported in figure 5 , the scale reporting values in units of $\mathrm{pSv} / e^{-}$.

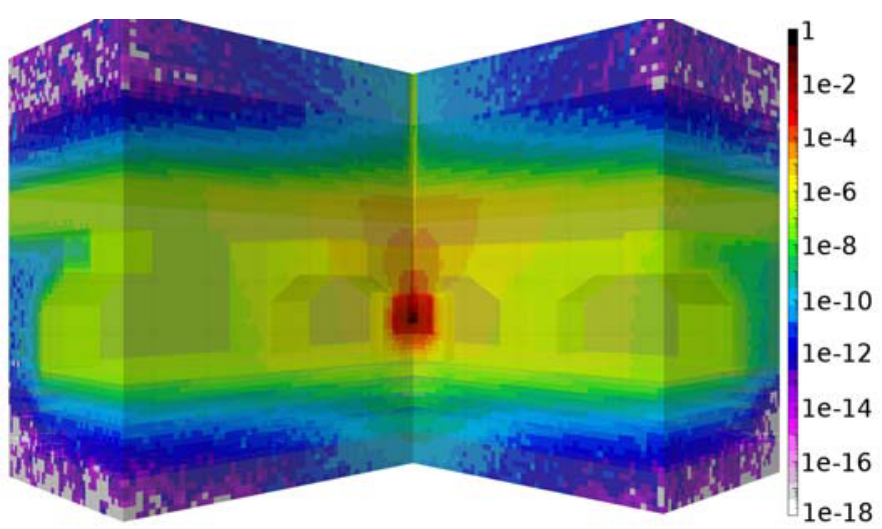

Figure 5. Prompt radiation ambient dose equivalent rate 3D map (clipped view) for the $320 \mathrm{MeV}$ beam dump. Values are reported in units of $\mathrm{pSv} / e^{-}$.

The soil and ground-water beyond the concrete basement, as modeled in our simulations, receive an averaged $\mathrm{H}^{*}(10)$ rate of $\approx 1.6 \mu \mathrm{Sv} / \mathrm{h}$, as reported in figure 6 . The average is taken over the single bins the indicated dose rate scoring volume was divided into. As a first result it fol- 
Table 1. Radionuclides found in air and their specific activities after $3000 \mathrm{hs}$ of beam dump irradiation, at $1 \mathrm{~h}$ cooling time. Acronyms PSN and PR stay for Parent Stable Nuclide and Production reaction, respectively.

\begin{tabular}{cccccc}
\hline Isotope & $\mathrm{A}$ & $\mathrm{PSN}$ & $\mathrm{PR}$ & $T_{1 / 2}$ & $A_{S}\left[\frac{\mathrm{Bq}}{\mathrm{cm}^{3}}\right]$ \\
\hline \hline $\mathrm{Ar}$ & 41 & ${ }^{40} \mathrm{Ar}$ & $(n, \gamma)$ & $1.8 \mathrm{~h}$ & $8.46 \cdot 10^{-3}$ \\
$\mathrm{Ar}$ & 37 & ${ }^{36} \mathrm{Ar}$ & $(n, \gamma)$ & $35.1 \mathrm{~d}$ & $2.70 \cdot 10^{-4}$ \\
$\mathrm{C}$ & 14 & ${ }^{14} \mathrm{~N}$ & $(n, p)$ & $5730 \mathrm{y}$ & $2.35 \cdot 10^{-4}$ \\
$\mathrm{H}$ & 3 & ${ }^{14} \mathrm{~N}$ & $\left(\gamma,{ }^{3} \mathrm{H}\right)$ & $12.3 \mathrm{y}$ & $4.96 \cdot 10^{-7}$ \\
$\mathrm{Ar}$ & 39 & ${ }^{38} \mathrm{Ar}$ & $(n, \gamma)$ & $269 \mathrm{y}$ & $1.21 \cdot 10^{-8}$ \\
\hline
\end{tabular}

lows that the prompt radiation field does not represent an issue from the point of view of the environmental impact.

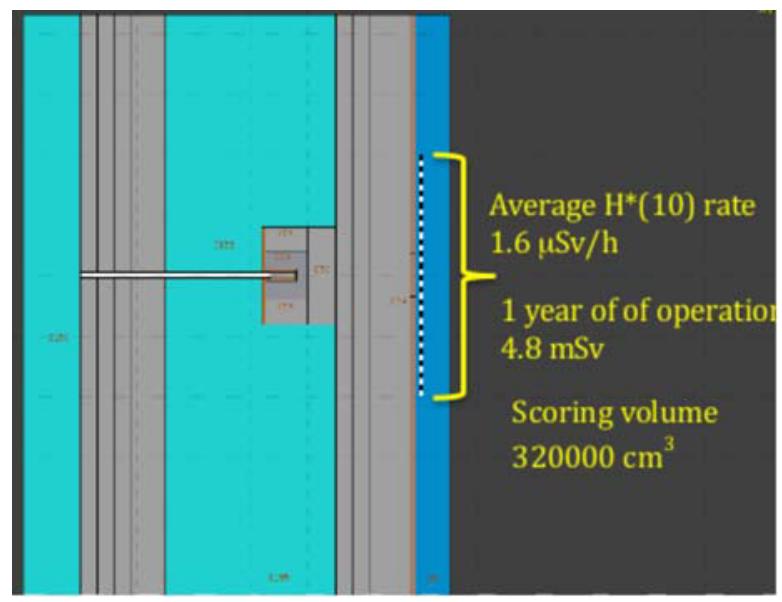

Figure 6. Average $\mathrm{H}^{*}(10)$ rate delivered to soil and ground-water by the prompt radiation generated at low energy dump level.

\subsection{Residual dose rates}

Residual dose rates in soil and ground-water were found to be trivial and are not further addressed here. Special attention was paid on the estimation of the air activation in the dump area, both because there is and there could be no ventilation system and of the risk assessment for personnel in case of emergency access in the dump proximities. In our simulations we asked FLUKA to score the produced radionuclides and their volume specific activities in air, after an irradiation of $3000 \mathrm{~h}$ (equivalent to one year of full machine runtime) and at several cooling times, namely $1 / 2 \mathrm{~h}, 1 \mathrm{~h}, 4 \mathrm{~h}, 8 \mathrm{~h}, 24 \mathrm{~h}$. Just as an example we report in the table 1 the radionuclides scored and their specific activities at $1 \mathrm{~h}$ cooling time. It is clear that several radionuclides commonly produced in air by $e^{-}$beams, like ${ }^{7} \mathrm{Be},{ }^{11} \mathrm{C},{ }^{13} \mathrm{~N},{ }^{15} \mathrm{O},{ }^{16} \mathrm{~N},{ }^{38} \mathrm{Cl},{ }^{39} \mathrm{Cl}$ were not scored in our simulations. These radionuclides are produced via photo-nuclear reactions with specific thresholds energies, exceeding $\approx 10 \mathrm{MeV}$ (actually the lowest threshold energy is $10.6 \mathrm{MeV}$ for the $(\gamma, n)$ reaction on ${ }^{14} \mathrm{~N}$, to produce $\left.{ }^{13} \mathrm{~N}\right)$. To establish the physical mechanisms justifying the absence of the above mentioned radionuclides in air in the dump region, the $\gamma$ fluence in air was computed, for one beam shot at nominal intensity as reported in section 1 , at $1 \mathrm{~s}$ and $1 / 2 \mathrm{~h}$ cooling times after $3000 \mathrm{~h}$ irradiation. The results are plotted in terms of isolethargic fluence, in figure 7. As it can be seen from the prompt radiation field

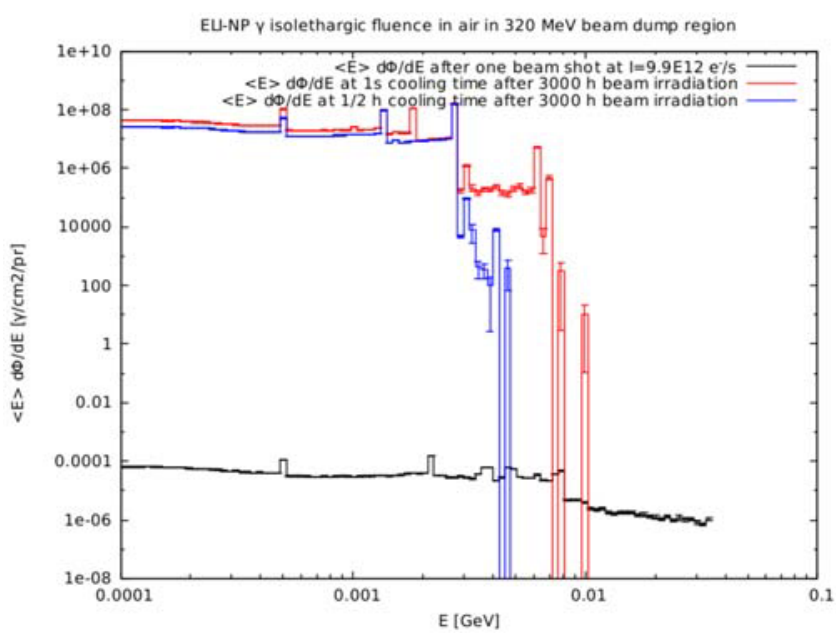

Figure 7. $\gamma$ isolethergic fluence in air in $320 \mathrm{MeV}$ beam dump area.

(that one which should lead to airborne activation) curve in black, very few photons $\left(\approx 10^{-6} \gamma / \mathrm{cm}^{2} / \mathrm{pr}\right)$ are scored above the threshold energy of $10.6 \mathrm{MeV}$, thus not sufficient to activate the radioisotopes of interest. For comparison, at $1 \mathrm{~s}$ cooling time and at $1 / 2 \mathrm{~h}$ cooling time all photons have energies well below that lowest threshold. That is why Be, $\mathrm{C}, \mathrm{N}, \mathrm{O}$ and $\mathrm{Cl}$ radioisotopes were not scored in our simulations. It is noteworthy to stress that the exemption level for ${ }^{41} \mathrm{Ar}$ in the Romanian law is $100 \mathrm{~Bq} / \mathrm{g}$.

In figure 8 the evolution of the residual $\mathrm{H}^{*}(10)$ rate at 1 $\mathrm{m}$ from the lateral dump surface and at $1 \mathrm{~m}$ height from the floor is plotted, as a function of the cooling times, while the same quantity spatial distributions at $1 / 2 \mathrm{~h}$ and $24 \mathrm{~h}$ are shown in figure 9 , with values in units of $\mathrm{pSv} / \mathrm{s}$. We can observe that already at $1 \mathrm{~s}$ after the end of irradiation an acceptable dose rate is found, $\approx 3 \mu \mathrm{Sv} / \mathrm{h}$, which decreases to $\approx 0.9 \mu \mathrm{Sv} / \mathrm{h}$ at $24 \mathrm{~h}$ of cooling time.

The ${ }^{41} \mathrm{Ar}$ contribution to the residual $\mathrm{H}^{*}(10)$ rate is found to be negligible, being $\sim 10^{-5} \mu \mathrm{Sv} / \mathrm{h}$. The $\mathrm{H}^{*}(10)$ rate due to the dump's materials and air activation, after the specified irradiation profile, at $1 \mathrm{~m}$ from the dump's surface, 5 minutes after machine shutdown, is $\approx 2.8 \mu \mathrm{Sv} / \mathrm{h}$. Thus $2.8 \mu \mathrm{Sv}$ is the upper bound effective dose a worker could receive if entering the area right after 5 minutes from the machine shutdown and resting at $1 \mathrm{~m}$ from the dump's surface for an hour. As a conclusion, the air activation radiation hazard in the low energy dump area was found to be negligible.

\section{High energy 840 MeV beam dump}

The high energy $740 \mathrm{MeV}$ ( $840 \mathrm{MeV}$ ) dump will be used to absorb the $e^{-}$beam during the machine operation in the high energy $\gamma$ beam production mode. The complete 3D 

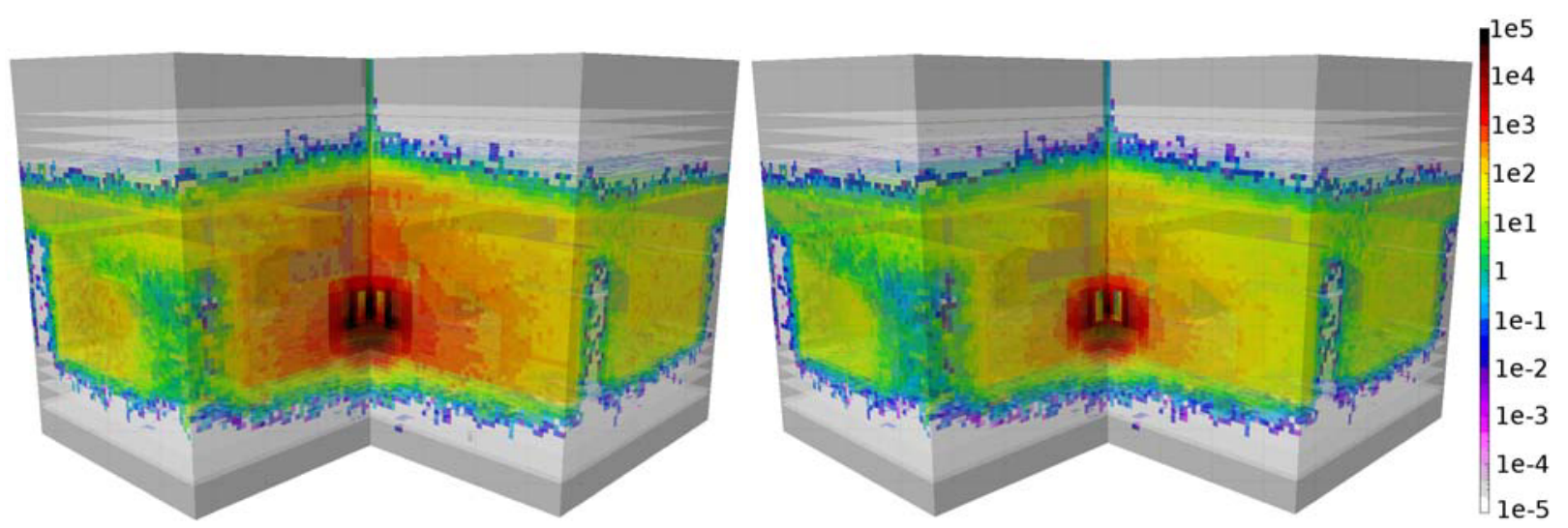

Figure 9. Low energy dump residual $\mathrm{H}^{*}(10)$ rate 3D maps (clipped views), at $1 / 2 \mathrm{~h}$ (left) and $24 \mathrm{~h}$ (right) cooling times after $3000 \mathrm{~h}$ beam dump irradiation. Scale values are in units of $\mathrm{pSv} / \mathrm{s}$.

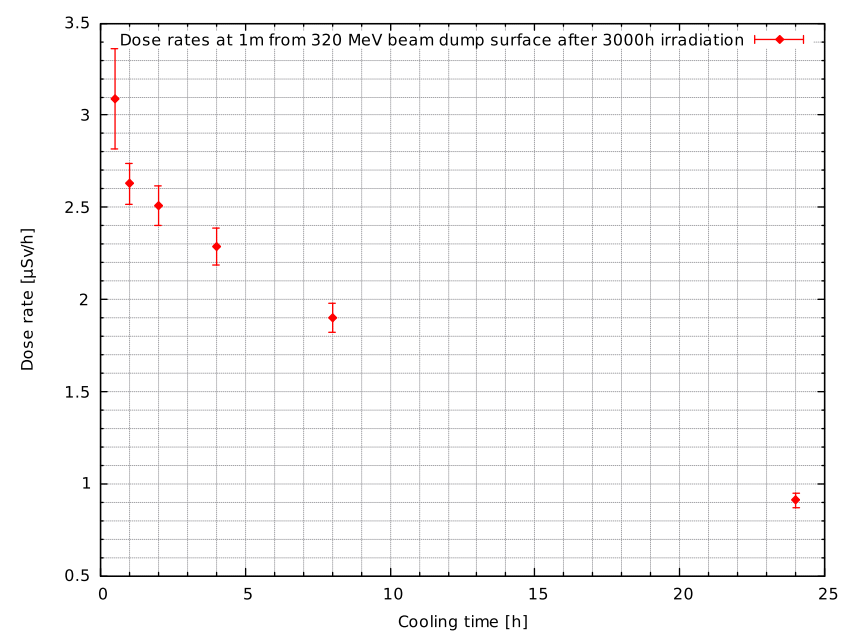

Figure 8. Low energy dump residual $\mathrm{H}^{*}(10)$ rate at $1 \mathrm{~m}$ from the dump lateral surface, at $1 \mathrm{~m}$ height from the floor, after $3000 \mathrm{~h}$ of beam dump irradiation, as a function of cooling times.

models' geometry, as simulated in FLUKA, for the detailed position and structure of the dump are illustrated in figure 10 and 11, respectively. For this dump the simulations' assumptions are:

- $\mathrm{E}\left(e^{-}\right)=840 \mathrm{MeV}$

- $\mathrm{I}=1.6 \mu \mathrm{A} \approx 9.9 \cdot 10^{12} e^{-} / \mathrm{s}$

- Machine runtime $=3000 \mathrm{~h} / \mathrm{y}$.

Despite the low energy one, the high energy dump does benefit of a dedicated hosting room, as indicated in figure 1, called Electron Recovery Area (ERA). The dump features a geometry concept similar to the low energy one, with slight differences due to the horizontal and suspended positioning in the ERA. This allows for the thermal cooling system to be arranged quite differently, with the internal copper cladding terminating on the radiator cap which is placed at the end of the dump, along the beam direction. From the internal structure point of view, the dump is modeled as a cylinder of $160 \mathrm{~cm}$ in diameter and 200 $\mathrm{cm}$ in height, with inner core cylinders of ordinary con-

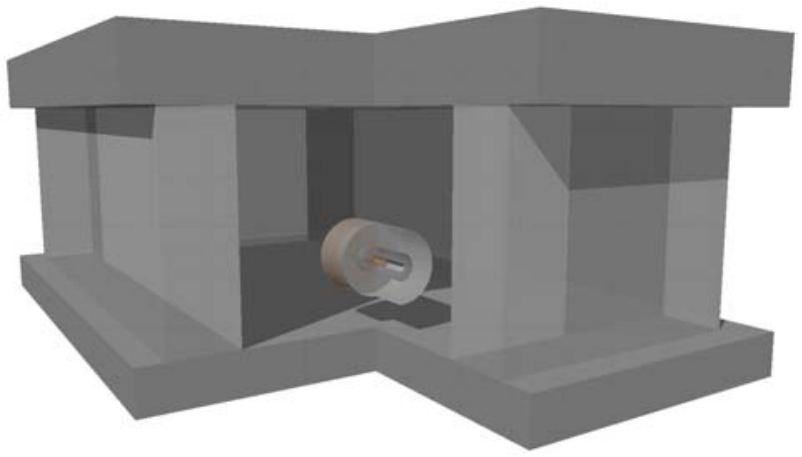

Figure 10. FLUKA 3D model (clipped view) for the position and orientation of the $840 \mathrm{MeV}$ beam dump in the dedicated host room, complete with the building shielding walls.

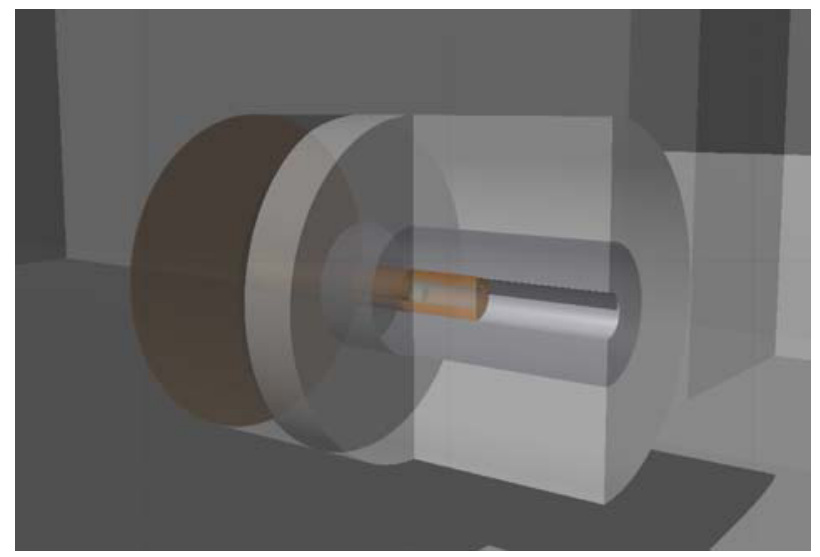

Figure 11. FLUKA 3D model (clipped view) for the structural details of the $840 \mathrm{MeV}$ beam dump.

crete, tungsten and lead, sorrounded by lead and ordinary concrete.

\subsection{Prompt radiation field}

As in the case of the low energy beam dump, the interaction of the $e^{-}$beam with dumps' materials does produce 
secondary and tertiary radiation fields composed mainly by neutrons and photons. Prompt radiation ambient dose equivalent rate 3D map is reported in figure 12 for the 840 $\mathrm{MeV}$ dump. The $\mathrm{H}^{*}(10)$ rate values, due to the prompt radiation field, found at $1 \mathrm{~m}$ from the dump's surface and at beam pipe level (dump longitudinal axis) are reported in the histogram of figure 13. Here we can see how the backscattered radiation determines a very high dose rate value of more then $1 \mathrm{~Sv} / \mathrm{h}$. While the dose level in the forward direction is the lower one, as expected, of $\approx 7$ $\mathrm{mSv} / \mathrm{h}$, the levels on the left and right do access to $\approx 30$ $\mathrm{mSv} / \mathrm{h}$. Moreover, in figure 14 the binned scoring re-

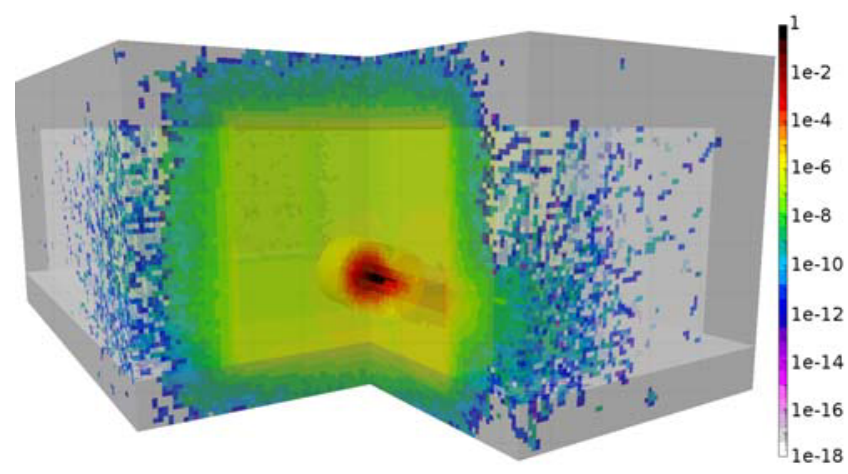

Figure 12. Prompt radiation ambient dose equivalent rate $3 \mathrm{D}$ map for the $840 \mathrm{MeV}$ beam dump.

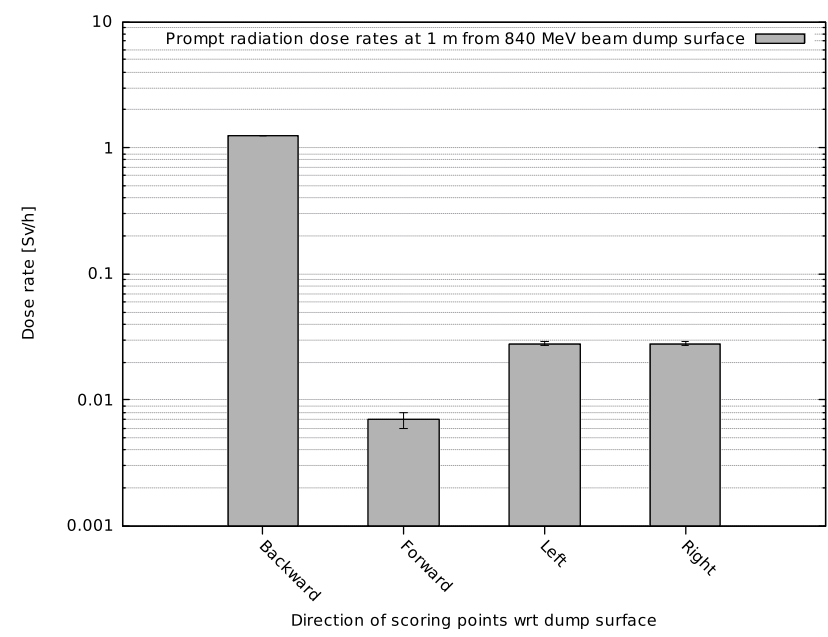

Figure 13. Ambient dose equivalent rates at $1 \mathrm{~m}$ from the dump's surface.

gions of $\mathrm{H}^{*}(10)$ outside the shielding walls (long pencils in black and white) and at $1 \mathrm{~m}$ from the dump's surface (white spots) are depicted, in overlay to the simulated geometry.

The $\mathrm{H}^{*}(10)$ rate mean values, due to the prompt radiation, in the scoring volumes outside the ERA shielding walls, in the forward and lateral directions with respect to the beam direction (referring to figure 14 right and downside pencils, respectively), were found to be $0.67 \mu \mathrm{Sv} / \mathrm{h}$ and $0.13 \mu \mathrm{Sv} / \mathrm{h}$ respectively, both well below the $1.4 \mu \mathrm{Sv} / \mathrm{h}$ constraint mentioned in section 1 . For the other lateral

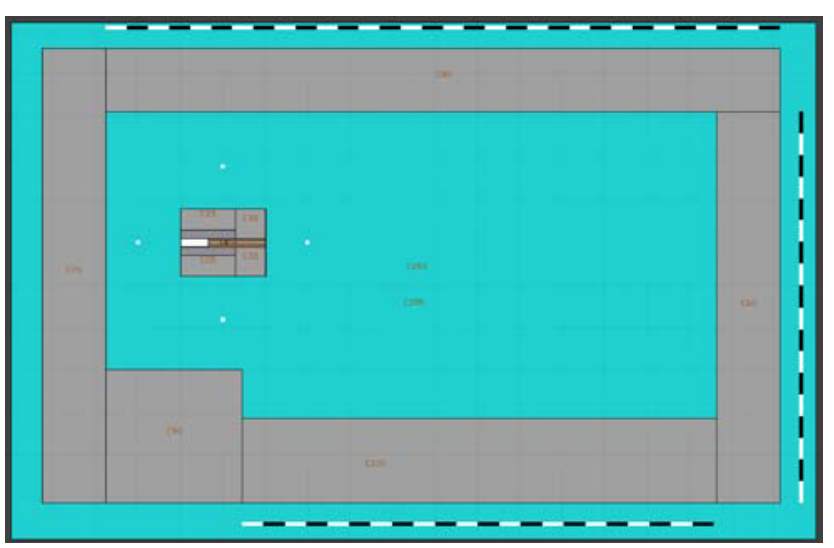

Figure 14. ERA FLUKA model, with the binned regions for the scoring of $\mathrm{H}^{*}(10)$ (the pencils along the lateral shielding walls and the four spots at $1 \mathrm{~m}$ from the beam surface).

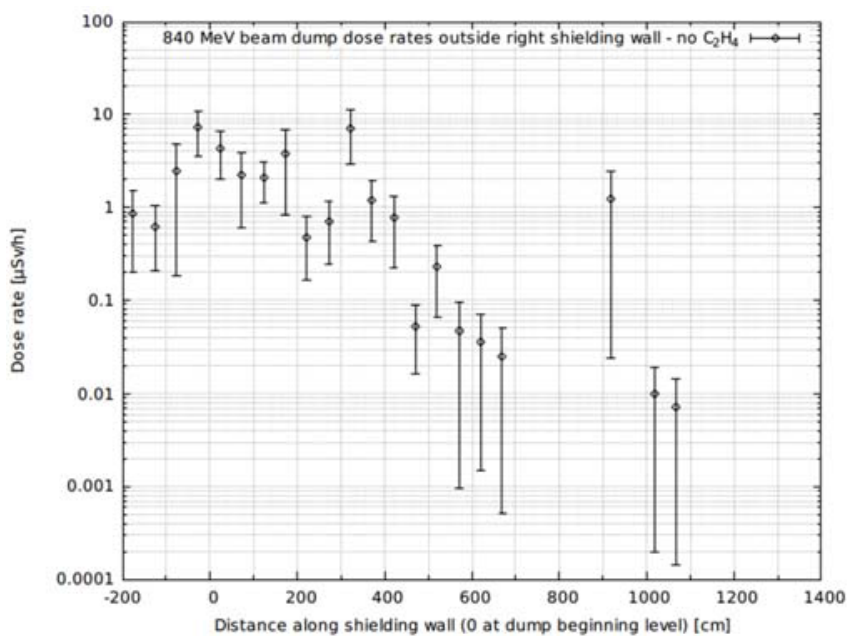

Figure 15. Ambient dose equivalent rates out of the lateral shielding wall (toward GP04 room), as a function of the distance along the wall.

shielding wall (upside in figure 14 ), the $\mathrm{H}^{*}(10)$ rate as a function of the distance along the wall is plotted in figure 15 . Here it is clearly visible that some values reach $\approx 7 \mu \mathrm{Sv} / \mathrm{h}$, which obviously was not acceptable in view of the constraints discussed above. Taking into account that the dominant contribution to the prompt radiation field in this region is due to neutrons, an asymmetric $25 \mathrm{~cm}$ thick $\left(\mathrm{C}_{2} \mathrm{H}_{4}\right)_{n}$ shell was added, as in figure 16. This countermeasure allowed a reduction of the scored dose rate by more than a factor of 10 at the dump beginning level, from $7 \mu \mathrm{Sv} / \mathrm{h}$ to $0.14 \mu \mathrm{Sv} / \mathrm{h}$, thus allowing us to be compliant with the dose rate constraints.

\subsection{Residual dose rates}

The specific activities of the scored radionuclides in air in the ERA room are reported in table 2, for $3000 \mathrm{~h}$ of beam dump irradiation (equivalent to one year of full machine 

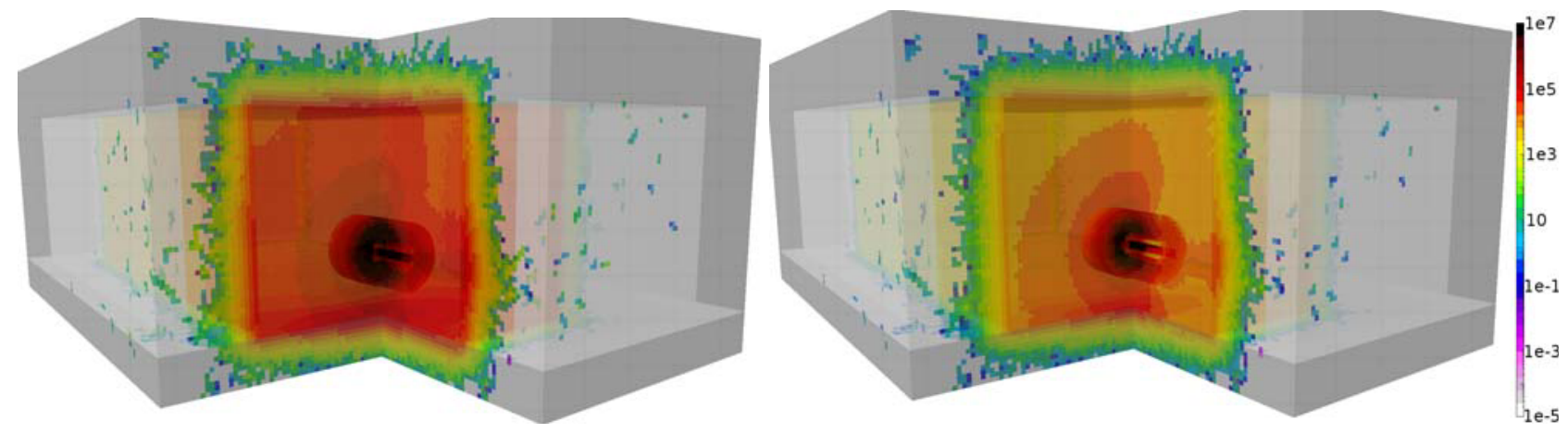

Figure 17. High energy dump residual $\mathrm{H}^{*}(10)$ rate 3D maps (clipped views), at $1 \mathrm{~s}$ (left) and $24 \mathrm{~h}$ (right) cooling times after $3000 \mathrm{~h}$ beam dump irradiation. Scale values are in units of $\mathrm{pSv} / \mathrm{s}$.

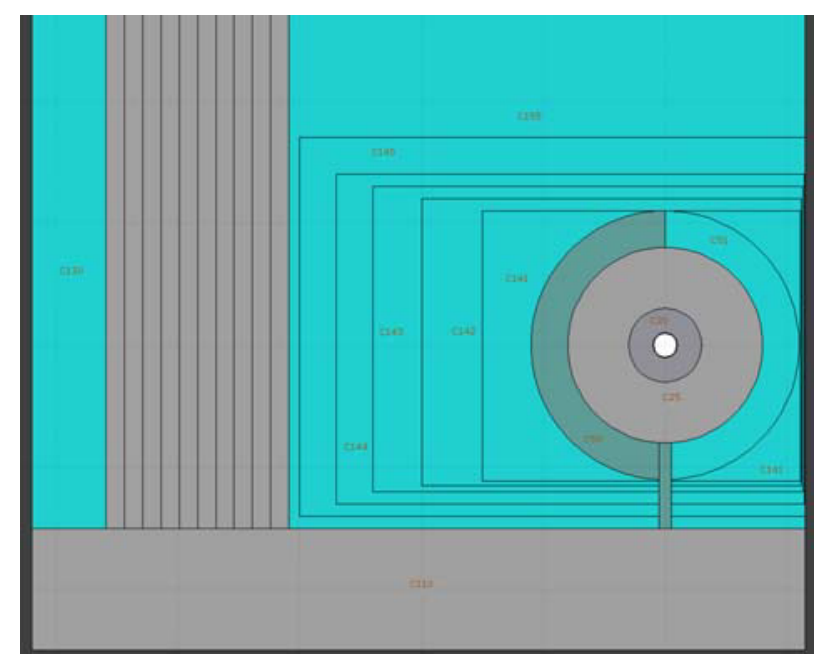

Figure 16. Asymmetric $\mathrm{C}_{2} \mathrm{H}_{4}$ shield geometry for the $840 \mathrm{MeV}$ beam dump. The concentric rectangles around the dump are the used biasing regions for this set of simulations.

runtime) and at $1 \mathrm{~h}$ of cooling time. Here we can find some of the above-mentioned radioisotopes commonly found at $e^{-}$accelerators, like ${ }^{13} \mathrm{~N}$ and ${ }^{15} \mathrm{O}$ not scored in the low energy dump case, with specific activities more than 3 and 11 orders of magnitude, respectively, lower then the maximum scored one, that of ${ }^{41} \mathrm{Ar}$.

In the case of high energy dump hosted in ERA room, the potential access by the personnel after the end of the irradiation is taken into account not for the emergency only, as it was for the low energy dump, but also for normal mantainance works. A ventilation system is thus foreseen for this area. In figure 17 the spatial distributions of $\mathrm{H}^{*}(10)$ rate are shown, for $1 \mathrm{~s}$ and $24 \mathrm{~h}$ cooling times, with reported values in units of $\mathrm{pSv} / \mathrm{s}$. Consequently, the ambient dose equivalent rates, due to residual radioactivity, as a function of the cooling times, at $1 \mathrm{~m}$ from the dump's surface in the four directions are reported in the plot of figure 18. With respect to the low energy dump case, we can observe rather high residual dose rates in all directions (the lateral ones being the higher ones), still at $24 \mathrm{~h}$ after the end of irradiation. At $1 \mathrm{~m}$ from the dump's surface, on the left and on the right, indeed, residual $\mathrm{H}^{*}(10)$ rate goes

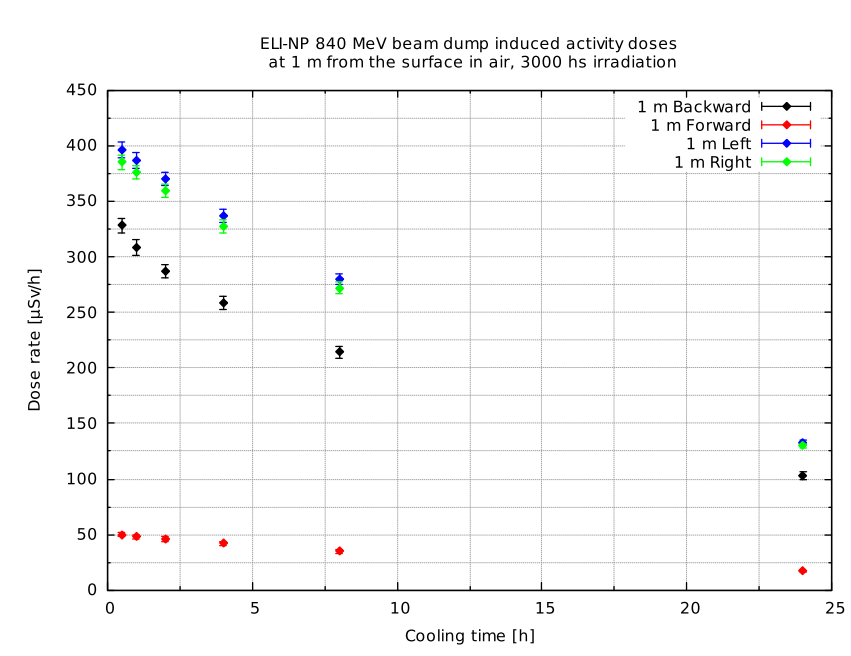

Figure 18. High energy dump residual $\mathrm{H}^{*}(10)$ rate at $1 \mathrm{~m}$ from the dump's surface, after $3000 \mathrm{~h}$ of beam dump irradiation, as a function of cooling times.

from $\approx 400 \mu \mathrm{Sv} / \mathrm{h}$ at $1 \mathrm{~s}$ of cooling time to $\approx 130 \mu \mathrm{Sv} / \mathrm{h}$ at $24 \mathrm{~h}$ of cooling time. The increase in the radiation hazard for personnel for this dump area, however, is strongly mitigated considering that working personnel could access in ERA only for a limited amount of time and, moreover, the presence of the ventilation system will operate in order to lower the intake risk from airborne activation. Under these circumstances no criticalities are advised from the radiological safety point of view.

Table 2. Radionuclides found in air in the ERA room and their specific activities after $3000 \mathrm{~h}$ of beam dump irradiation, at $1 \mathrm{~h}$ cooling time. Acronyms do stay for the same as in table 1 .

\begin{tabular}{cccccc}
\hline Isotope & $\mathrm{A}$ & $\mathrm{PSN}$ & $\mathrm{PR}$ & $T_{1 / 2}$ & $A_{S}\left[\frac{\mathrm{Bq}}{\mathrm{cm}^{3}}\right]$ \\
\hline \hline $\mathrm{Ar}$ & 41 & ${ }^{40} \mathrm{Ar}$ & $(n, \gamma)$ & $1.83 \mathrm{~h}$ & $7.32 \cdot 10^{-3}$ \\
$\mathrm{Ar}$ & 37 & ${ }^{36} \mathrm{Ar}$ & $(n, \gamma)$ & $35.1 \mathrm{~d}$ & $2.27 \cdot 10^{-4}$ \\
$\mathrm{C}$ & 14 & ${ }^{14} \mathrm{~N}$ & $(n, p)$ & $5730 \mathrm{y}$ & $2.17 \cdot 10^{-4}$ \\
$\mathrm{H}$ & 3 & ${ }^{14} \mathrm{~N}$ & $\left(\gamma,{ }^{3} \mathrm{H}\right)$ & $12.3 \mathrm{y}$ & $5.66 \cdot 10^{-6}$ \\
$\mathrm{~N}$ & 13 & ${ }^{14} \mathrm{~N}$ & $(\gamma, n)$ & $6.9 \mathrm{~d}$ & $1.84 \cdot 10^{-6}$ \\
$\mathrm{Ar}$ & 39 & ${ }^{38} \mathrm{Ar}$ & $(n, \gamma)$ & $269 \mathrm{y}$ & $3.00 \cdot 10^{-8}$ \\
$\mathrm{O}$ & 15 & ${ }^{16} \mathrm{O}$ & $(\gamma, n)$ & $122.24 \mathrm{~s}$ & $7.43 \cdot 10^{-14}$ \\
\hline
\end{tabular}




\section{Conclusions}

All Monte Carlo calculations, performed by means of the FLUKA code under conservative conditions at maximum energy of $320 \mathrm{MeV}$ and $840 \mathrm{MeV}$, with beam current of $9.9 \cdot 10^{12} e^{-} / s$, and $3000 \mathrm{~h} / \mathrm{y}$ of machine operation are shown to be compliant with the dose constraints imposed by the Romanian law in force in radiation protection subject matter. The computed reference parameters $\left(\mathrm{H}^{*}(10)\right.$ rates, induced activity and radionuclides specific activities) for the dump in the basement area show that its environmental impact is trivial (i.e. negligible $\mathrm{H}^{*}(10)$ rate delivered to soil and water and negligible activations of both materials). The air activation also, in the basement area, was found to be not a critical issue. Our simulations' results show, also, that the high energy dump is conceived in a proper way to satisfy the dose limit constraint to the members of the public, outside the building shield. The dose levels scored at $50 \mathrm{~cm}$ far away from the shielding wall toward the GP04 room, however, were not acceptable in terms of the constraints specified in section 1. As a countermeasure we modeled the dump with an additional asymmetric $25 \mathrm{~cm}$ thick $\left(\mathrm{C}_{2} \mathrm{H}_{4}\right)_{n}$ shell on its lateral surface, what resulted to be an effective remedy which allowed the dump to be compliant with the dose constraints. The studied models have already passed the engineering phase and are presently in the construction process.

\section{References}

[1] N. V. Zamfir, "Extreme Light Infrastructure Nuclear Physics (ELI-NP): Present status and perspectives", EPJ Web of Conferences 117, 10001 (2016)

[2] Guvernul Romaniei, Cancelaria Primului Ministru, CNCAN, "Norme de Securitate Radiologica - Proceduri de Acceptare a Intraprinderilor Externe", NSR-09, (2003)

[3] I. O. Mitu, C. Ivan, F. Negoita, D. Aranghel, S. Bercea, E. Iliescu, C. Petcu, M. Gugiu, M. A. Popovici, C. A. Ur, S. Gales, N. V. Zamfir, "Radiation Protection and Safety at ELI-NP", Romanian Reports in Physics, 68 Supplement, S885-S945 (2016)

[4] A. Ferrari, P.R. Sala, A. Fasso', and J. Ranft, "FLUKA: a multi-particle transport code", CERN 2005-10 (2005), INFN/TC-05/11, SLAC-R-773

[5] T.T. Bohlen, F. Cerutti, M.P.W. Chin, A. Fasso', A. Ferrari, P.G. Ortega, A. Mairani, P.R. Sala, G. Smirnov, and V. Vlachoudis, "The FLUKA Code: Developments and Challenges for High Energy and Medical Applications", Nuclear Data Sheets 120, 211-214 (2014)

[6] O. Frasciello, unpublished results 